\title{
Singularity Confinement and Algebraic Integrability
}

\author{
S. LAFORTUNE* and A. GorielY ${ }^{\dagger}$ \\ Department of Mathematics \\ University of Arizona, \\ Tucson, AZ, 85721-0089, USA
}

June 19, 2018

\begin{abstract}
Two important notions of integrability for discrete mappings are algebraic integrability and singularity confinement, have been used for discrete mappings. Algebraic integrability is related to the existence of sufficiently many conserved quantities whereas singularity confinement is associated with the local analysis of singularities. In this paper, the relationship between these two notions is explored for birational autonomous mappings. Two types of results are obtained: first, algebraically integrable mappings are shown to have the singularity confinement property. Second, a proof of the non-existence of algebraic conserved quantities of discrete systems based on the lack of confinement property is given.
\end{abstract}

\section{INTRODUCTION}

One of the first notion of integrability was introduced by Liouville in the nineteenth century for Hamiltonian systems in classical mechanics. This type of integrability is based on quantities whose value do not change in time, the so-called constants of the motion or first integrals. For instance, in many mechanical systems the total energy and the linear and angular momenta are conserved through the dynamics and are examples of such constants. Liouville's fundamental contribution was to prove that if a given Hamiltonian systems admits enough first integrals, it can be solved explicitly by quadratures. More precisely, a $2 n$-dimensional Hamiltonian system is said to be Liouville-integrable if it admits $n$ functionally independent first integrals in involution (that is their Poisson Brackets commute) and such systems can be integrated by quadrature and enjoy a particularly simple topology (the flow lives on products of tori and cylinders). For systems of $n$ first-order ordinary differential equations (ODEs) algebraic integrability is defined as the existence $(n-1)$ functionally independent first integrals that are algebraic functions of the dependent variables. The existence of such first integrals is important for integrability as they can be used to reduce the dimensionality of the system.

In the particular case of Hamiltonian systems, algebraic integrability coincides with the notion of superintegrability. Indeed, for a $2 n$-dimensional Hamiltonian system, the $2 n-1$ first integrals needed for the property of algebraic integrability are much more than the $n$ ones needed for complete Liouville-Arnold integrability.

A different notion of integrability was introduced by Painlevé in the beginning of the 20th century 21, 5. Although the aim of Painlevé was not to define a notion of integrability but rather to build new functions, his property is today widely used for the detection of integrable systems. An ODE is said to possess the Painlevé property [1, 17,6] if its general solution is single-valued in its maximal domain of analytic continuation. The restrictions the Painlevé property impose on the solutions are so strong that an ODE exhibiting it may be considered for all practical purpose integrable. Many

*lafortus@math.arizona.edu

†'goriely@math.arizona.edu 
formal links between the Painlevé property and other notions of integrability have been established (see for example [6] 7]). Despite the fact that there is no general algorithmic way to obtain sufficient conditions for a given ODE to have the Painlevé property, necessary conditions can be derived. The algorithmic procedure to obtain such conditions is known as the Painlevé test or singularity analysis and is based on a local analysis of the solutions around movable isolated singularities.

The notions of algebraic integrability and singularity analysis of the solutions can be extended to finite difference equations. One of the most effective way to perform singularity analysis in this context is given by singularity confinement [7, 11, 12, 23, 4, 25, 26, 13, 14, 15, 16] which, like the Painlevé test for ODEs, imposes conditions on the singularities of the solutions. Although there exist many striking particular examples which indicate that singularity confinement is closely related to other notions of integrability, there is no clear-cut result which establishes this relationship in a formal and general framework. This paper explores the relationship between the notion of algebraic integrability and the property of singularity confinement in discrete mappings.

In the case of systems of ODEs, Yoshida [27 28,6] proved that the degree of a rational first integral is closely related to some exponents that can be obtained through the application of the Painlevé test. In this paper, we establish an equivalent result for discrete systems by showing that singularity confinement analysis provides necessary conditions for the existence of an algebraic first integral. Moreover, a lower bound on the degree of rational first integrals can be obtained.

The rest of this article is organized as follows. In Section I we define the notion of singularity and singularity confinement for birational autonomous discrete dynamical systems. In Section we study the local consequences of the existence of algebraic first integral on the confinement of the singularities in the two-dimensional case. In Section [IV] we extend the results of Sections [II] to arbitrary dimensions. Applications are discussed in Section $\mathrm{V}$

\section{FORMULATION OF THE PROBLEM}

In this section we introduce and illustrate the notion of singularity confinement by considering a particular class of two-dimensional autonomous dynamical systems. These examples are used to motivate a formal definition valid in arbitrary dimensions.

Throughout the article the following notation will be used: If $\mathbf{g}$ is an analytic function from $\mathbb{C}^{p}$ to $\mathbb{C}^{r}$, then $\operatorname{Dg}(\mathbf{x})$ is its Jacobian matrix evaluated at $\mathbf{x} \in \mathbb{C}^{p}$. When $p=r$, the Jacobian is denoted by $\operatorname{det}(\operatorname{Dg}(\mathbf{x}))$. If $f$ is a complex-valued rational function on $\mathbb{C}^{p}$, then $\operatorname{num}(f)$ and $\operatorname{den}(f)$ denote respectively the numerator and denominator of $f$.

\section{II.1 Simple examples}

The basic idea of singularity confinement is to consider the properties of solutions close to some singularities. To illustrate this concept, consider the class of two-dimensional complex dynamical systems of the form

$$
\left(\begin{array}{l}
x_{n+1} \\
y_{n+1}
\end{array}\right)=\mathbf{f}\left(x_{n}, y_{n}\right)=\left(\begin{array}{c}
g\left(x_{n}, y_{n}\right) \\
x_{n}
\end{array}\right)
$$

where $g$ is a complex-valued rational function on $\mathbb{C}^{2}$,

$$
g(x, y)=\frac{p(x, y)}{q(x, y)}
$$

and $p, q$ are relatively prime polynomials. Two types of singularities can be distinguished for such systems. The singularities of first type are the roots of $q$ in $\mathbb{C}^{2}$. That is the values $(x, y)$ at which the vector field $\mathbf{f}(x, y)$ in (2.1) has a singularity. The singularities of second type are the points where the Jacobian of $\mathbf{f}$ is zero (this type of singularities was first considered in [12]). As an example, consider the following discrete dynamical system:

$$
\left(\begin{array}{l}
x_{n+1} \\
y_{n+1}
\end{array}\right)=\mathbf{f}\left(x_{n}, y_{n}\right)=\left(\begin{array}{c}
-x_{n}-y_{n}+a+\frac{b}{x_{n}} \\
x_{n}
\end{array}\right),
$$


where $a$ and $b$ are complex numbers. The analysis is performed for generic values of the parameters $a$ and $b$. Clearly the right-hand-side of (2.2) presents a singularity if $x_{n}=0$. To study this singularity we study the behavior of nearby solutions by introducing a small complex perturbation $\epsilon$, with $|\epsilon|>0$. We then set $y_{0}$ to be any complex number and $x_{0}=\epsilon$. The iterates $x_{p}$ and $y_{p}$ are then determined for any positive nonzero integer $p$ by the discrete dynamical system (2.2). After one iteration, we have

$$
x_{1}=\frac{b}{\epsilon}+a-y_{0}+\mathcal{O}(\epsilon) .
$$

In the limit $\epsilon \rightarrow 0,\left|x_{1}\right| \rightarrow \infty$ and, using (2.2) again,

$$
\begin{aligned}
& x_{2}=-\frac{b}{\epsilon}+y_{0}+\mathcal{O}(\epsilon), \\
& x_{3}=-\epsilon+\mathcal{O}\left(\epsilon^{2}\right) .
\end{aligned}
$$

The limit $\epsilon \rightarrow 0$ of the next iterate $x_{4}$ is well defined and given by $y_{0}$. Despite the fact that the function f defined by (2.2) is not well defined on any point of $\mathbb{C}^{2}$ of the form $(0, y)$, the limit $(x, y) \rightarrow(0, y)$ of $\mathbf{f}^{4}(x, y)$ exists and is given by

$$
\lim _{(x, y) \rightarrow(0, y)} \mathbf{f}^{4}(x, y)=\left(\begin{array}{l}
y \\
0
\end{array}\right) .
$$

Moreover, since the value of the limit (2.6) depends on $y$, the same limit applied on the Jacobian of $\mathbf{f}^{4}$ is nonzero. The singularity is thus "confined" between the iterates 0 and 4 and does not propagate further. This property of confinement is very particular and we show in this paper that it is closely related the existence of a first integral for the system. A first integral for a discrete dynamical system of the form (2.1) is defined to be an analytic non-constant complex-valued function $I$ defined almost everywhere on $\mathbb{C}^{2}$ which is preserved by $\mathbf{f}$, that is

$$
I(\mathbf{f}(\mathbf{x}))=I(\mathbf{x}),
$$

for every $\mathbf{x} \in \mathbb{C}^{2}$ for which (2.77) makes sense. It is important to note that if $\mathbf{f}$ is not defined at a point $\mathbf{x}^{*}$ where $I$ is well-defined, then the value of $I$ at $\mathbf{x}^{*}$ is still preserved under $\mathbf{f}$ in the limit sense, i.e.

$$
\lim _{\mathbf{x} \rightarrow \mathbf{x}^{*}} I(\mathbf{f}(\mathbf{x}))=I\left(\mathbf{x}^{*}\right) .
$$

This is a consequence of the fact that $\mathbf{f}$ and $I$ are continuous and defined almost everywhere in $\mathbb{C}^{2}$. In the particular case of system (2.2), one can check that the following polynomial

$$
I(x, y)=x y(x+y-a)-b(x+y)
$$

is a first integral. Moreover, following (2.8), the first integral $I$ is preserved in the limit sense through the iterations of the singularity of (2.2). That is,

$$
-b y=I(0, y)=\lim _{(x, y) \rightarrow(0, y)} I \circ\left(\mathbf{f}^{i}(x, y)\right), \quad i=1,2,3,4 .
$$

Note that most integrable systems used in this paper are particular cases of the so-called QRT-family of mappings [22]15].

The confinement property for the solutions of (2.1) does not hold in general. Although there is no formal result establishing how rare this confinement property might be, the fact that it does not hold for most systems of the form (2.1) has been well established in the literature 11, 23, 25, 26, 13, 14 .

The following system is an example where singularities are not confined:

$$
\left(\begin{array}{l}
x_{n+1} \\
y_{n+1}
\end{array}\right)=\mathbf{f}\left(x_{n}, y_{n}\right)=\left(\begin{array}{c}
-x_{n}-y_{n}+a+\frac{b}{x_{n}^{3}} \\
x_{n}
\end{array}\right) .
$$

Consider the singularity $\left(x^{*}, y^{*}\right)=(0, y)$ and, as before, introduce $x_{0}=\epsilon$ and $y_{0} \in \mathbb{C}$ nonvanishing. The next iterates are $x_{1}=b / \epsilon^{3}+\left(a-y_{0}\right)-\epsilon, x_{2}=-b / \epsilon^{3}+y_{0}+\mathcal{O}\left(\epsilon^{5}\right)$, and $x_{3}=\epsilon+\mathcal{O}\left(\epsilon^{5}\right)$. Further, 
we find that, unlike the previous example, the appropriate cancellations allowing for confinement do not occur and the next iterates are also diverging at $\epsilon=0, x_{4}=2 b / \epsilon^{3}+\left(a-y_{0}\right)-\epsilon+\mathcal{O}\left(\epsilon^{5}\right)$, and $x_{5}=-2 b / \epsilon^{3}+y_{0}+\mathcal{O}\left(\epsilon^{5}\right)$. In general, the sequence of limits $\epsilon \rightarrow 0$ of the $\left|x_{p}\right|$ for $p>0$ repeats the formal triplet $(\infty, \infty, 0)$ indefinitely. This assertion is proven by induction based on the equality

$$
\mathbf{f}^{3}\left(\begin{array}{l}
-n b / \epsilon^{3}+y_{0}+\mathcal{O}\left(\epsilon^{5}\right) \\
n b / \epsilon^{3}+\left(a-y_{0}\right)-\epsilon+\mathcal{O}\left(\epsilon^{5}\right)
\end{array}\right)=\left(\begin{array}{l}
-(n+1) b / \epsilon^{3}+y_{0}+\mathcal{O}\left(\epsilon^{5}\right) \\
(n+1) b / \epsilon^{3}+\left(a-y_{0}\right)-\epsilon+\mathcal{O}\left(\epsilon^{5}\right)
\end{array}\right)
$$

for any positive integer $n$. Therefore, the singularity is not confined. It is generally believed that systems which lack the confinement property will not be integrable. However, there is no definite result attached to this belief. We prove in the next sections that we can use the analysis of the dynamics nearby the singularities can be used to conclude that (2.11) does not admit an algebraic first integral.

The following example illustrates the occurrence of the second type of singularity. Consider

$$
\left(\begin{array}{l}
x_{n+1} \\
y_{n+1}
\end{array}\right)=\mathbf{f}\left(x_{n}, y_{n}\right)=\left(\begin{array}{c}
\frac{x_{n}-1}{y_{n}}+2 x_{n} \\
x_{n}
\end{array}\right) .
$$

At first sight, the point $\left(x_{n}, 0\right)$ seems to be the only singularity. However, the Jacobian of $\mathbf{f}$ in (2.13) vanishes whenever $x_{n}=1$. If $x_{0}=1$ and $y_{0} \neq 0$, the next iterate as determined by (2.13) is given by $x_{1}=2$. We then have $x_{2}=5, x_{3}=12$. It is not difficult to see that $x_{i}$ grows with respect to $i$ and that all iterates, of $(1, y)$ under (2.13) with $y \neq 0$ are independent of $y$. The singularity here only appears in the inverse of the Jacobian matrix and is not confined.

In the light of the previous examples, we can define the confinement property for singularities of discrete dynamical systems of the form (2.1). A general definition will be given in the next section. A singularity of the dynamical system (2.1) is defined to be any point $\left(x^{*}, y^{*}\right)$ in $\mathbb{C}^{2}$ at which the right-hand-side of (2.1) is undefined or at which the Jacobian of $\mathbf{f}$ is zero. A singularity $\left(x^{*}, y^{*}\right)$ is said to be confined if there exists a positive integer $N$ such that both limits

$$
\lim _{(x, y) \rightarrow\left(x^{*}, y^{*}\right)} \mathbf{f}^{N}(x, y), \quad \lim _{(x, y) \rightarrow\left(x^{*}, y^{*}\right)} \operatorname{det}\left(\operatorname{Df}^{N}(x, y)\right)
$$

exist and the second limit is nonzero. The smallest number $N$ having this property is referred to as the confinement number, that is the number of steps necessary for confinement.

The next example illustrates the case when a singularity of the second type is confined.

$$
\left(\begin{array}{c}
x_{n+1} \\
y_{n+1}
\end{array}\right)=\mathbf{f}\left(x_{n}, y_{n}\right)=\left(\frac{\left(x_{n}-1 / a\right)\left(x_{n}-a\right)}{y_{n}\left(x_{n}-b\right)\left(x_{n}-1 / b\right)}\right)
$$

where $a$ and $b$ are two nonzero distinct complex numbers which are also both distinct from $1 / a$ and $1 / b$. The right-hand side (RHS) of (2.15) presents a singularity of the second type if $x_{n}=a$ because the Jacobian of $\mathbf{f}$ vanishes. If $x_{0}=a$ and $y_{0} \neq 0$, the next iterate as determined by 2.15 will be $x_{1}=0$, then $x_{2}=1 / a$ and $x_{3}$ takes an indeterminate form $0 / 0$. Again, we introduce a small complex perturbation $\epsilon,|\epsilon|>0$, then set $x_{0}=\epsilon$ to find that when $\epsilon \rightarrow 0$,

$$
x_{3} \rightarrow y_{0}+a-\frac{1}{a},
$$

and we conclude that the singularity is confined. The dynamical system 2.15) has another singularity of the first type if $x_{n}=b$. Applying the same procedure as before with a perturbation $\epsilon$, one finds that the limit $\epsilon \rightarrow 0$ of $\left|x_{1}\right|, x_{2}$ and $x_{3}$ gives, respectively, $\infty, 1 / b$ and $b y_{0} /\left(b+y_{0}\left(1-b^{2}\right)\right)$. Thus, any singularity of the form $\left(b, y_{0}\right)$ with $y_{0} \neq b /\left(b^{2}-1\right)$ is confined in 3 steps. System (2.15) admits the following rational first integral

$$
I(x, y)=\left(b+\frac{1}{b}\right)(x+y)+\left(a+\frac{1}{a}\right)\left(\frac{1}{x}+\frac{1}{y}\right)-\frac{\left(1+x^{2}\right)\left(1+y^{2}\right)}{x y} .
$$


The following example shows that the existence of a first integral does not imply that all singularities are confined. The system

$$
\left(\begin{array}{c}
x_{n+1} \\
y_{n+1}
\end{array}\right)=\mathbf{f}\left(x_{n}, y_{n}\right)=\left(\begin{array}{c}
x_{n}^{2} / y_{n} \\
x_{n}
\end{array}\right),
$$

has the following first integral

$$
I(x, y)=\frac{x y}{x^{2}+y^{2}} .
$$

The RHS of (2.18) has a pole of order 1 at any point of $\mathbb{C}^{2}$ of the form $(x, 0)$ with $x \neq 0$. As before, if we study this singularity by perturbation we conclude that there is no confinement since the Laurent expansions of the iterations of $(x, \epsilon)$ under the discrete system (2.18) around $\epsilon=0$ are all divergent. Although this singularity is not confined, one notices that any point of the form $(x, 0)$ with $x \neq 0$ does not have a unique preimage under (2.18). Indeed, the expression defining $\mathbf{f}^{-1}$ given by

$$
\mathbf{f}^{-1}(x, y)=\left(\begin{array}{c}
y \\
y^{2} / x
\end{array}\right)
$$

has a singularity of second type at $(x, 0)$ and all the points of this form are mapped to $(0,0)$ under $\mathbf{f}^{-1}$. This example shows that if one wishes to establish a relationship between singularity confinement and first integrals, particular care should be given in distinguishing different singularities.

\section{II.2 Preliminaries}

The notion of singularity confinement will now be defined in a more general setting. We consider $p$-dimensional autonomous discrete dynamical systems described by birational mappings on $\mathbb{C}^{p}$. A birational mapping on $\mathbb{C}^{p}$ is a $\mathbb{C}^{p}$-valued rational function which is one-to-one almost everywhere in $\mathbb{C}^{p}$ and whose inverse, where it exists, is also represented by a rational function. The corresponding discrete dynamical systems associated are written:

$$
\mathbf{x}_{n+1}=\mathbf{f}\left(\mathbf{x}_{n}\right), \quad \mathbf{x}_{n} \in \mathbb{C}^{p}, \quad n \in \mathbb{Z},
$$

where $\mathbf{f}$ is a birational mapping on $\mathbb{C}^{p}$ with no explicit dependence on $n$. The ith iterate of $\mathbf{x}_{0} \in \mathbb{C}^{p}$ under (2.21) is said to exist if the following limit

$$
\lim _{\mathbf{x} \rightarrow \mathbf{x}_{0}} \mathbf{f}^{i}(\mathbf{x})
$$

exists. The $i$ th iterate is then defined to be that limit and is denoted $\mathbf{F}^{i}\left(\mathbf{x}_{0}\right)$.

Definition. A singularity of type I for the discrete dynamical system $\mathbf{x}_{n+1}=\mathbf{f}\left(\mathbf{x}_{n}\right)$ is a point $\mathbf{x}^{*} \in \mathbb{C}^{p}$ at which $\mathbf{f}$ is not defined. The set of singularity of type I associated with the mapping $\mathbf{f}$ in $\mathbb{C}^{p}$ is denoted $S_{I}(\mathbf{f})$.

Definition. A singularity of type II for the discrete dynamical system $\mathbf{x}_{n+1}=\mathbf{f}\left(\mathbf{x}_{n}\right)$ is a point $\mathbf{x}^{*} \in \mathbb{C}^{p}$ such that $\operatorname{det}\left(D \mathbf{f}\left(\mathbf{x}^{*}\right)\right)=0$. The set of singularity of type II in $\mathbb{C}^{p}$ is denoted $S_{I I}(\mathbf{f})$.

In the particular case of birational mappings, we have

$$
\begin{aligned}
S_{I}(\mathbf{f}) & =\left\{\mathbf{x}^{*} \in \mathbb{C}^{p} \mid \operatorname{den}\left(f_{i}\right)\left(\mathbf{x}^{*}\right)=0, \text { for some } i \in\{1,2, \ldots, p\}\right\}, \\
S_{I I}(\mathbf{f}) & =\left\{\mathbf{x}^{*} \in \mathbb{C}^{p} \mid \operatorname{det}\left(\operatorname{Df}\left(\mathbf{x}^{*}\right)\right)=0\right\} .
\end{aligned}
$$

We call the sets $S_{I}(\mathbf{f})$ and $S_{I I}(\mathbf{f})$, the singular sets of first and second type, respectively. The singular set $S(\mathbf{f})$ is defined as the union of both sets of first and second type

$$
S(\mathbf{f})=S_{I}(\mathbf{f}) \cup S_{I I}(\mathbf{f}) .
$$


For both types of singularity we can define the property of confinement.

Definition. Let $\mathbf{x}^{*} \in \mathbb{C}^{p}$ be a singularity of type I or II for the system $\mathbf{x}_{n+1}=\mathbf{f}\left(\mathbf{x}_{n}\right)$. The singularity is said to be confined if, for some $i_{c} \in \mathbb{N}^{>0}$, the iterate $\mathbf{F}^{i_{c}}\left(\mathbf{x}^{*}\right)$ exists and $\lim _{\mathbf{x} \rightarrow \mathbf{x}^{*}} \operatorname{det}\left(D \mathbf{f}^{i_{c}}(\mathbf{x})\right) \neq 0$.

The lowest such $i_{c}$ is referred to as the confinement number that is the number of steps necessary for confinement. From now on, we assume that $\mathbf{f}$ is a birational mapping. The following lemma proves to be useful.

Lemma 2.1. The image of $S_{I I}(\mathbf{f})$ under the mapping $\mathbf{f}$ lies inside an algebraic variety of codimension 2.

That is, the birational mapping $\mathbf{f}$ is one-to-one only on the subset of $\mathbb{C}^{p}$ defined by

$$
O_{\mathbf{f}} \equiv \mathbb{C}^{p} \backslash S(\mathbf{f}) .
$$

As a consequence, the function $\mathbf{f}$ restricted to $O_{\mathbf{f}}$ defines a rational diffeomorphism

$$
\mathbf{f}: O_{\mathbf{f}} \rightarrow O_{\mathbf{f}}^{\prime} \equiv \mathbf{f}\left(O_{\mathbf{f}}\right),
$$

whose inverse is also a rational function.

Proof. Assume that $S_{I I}(\mathbf{f}) \neq \emptyset$. Let $\mathbf{x}_{0} \in S_{I I}(\mathbf{f})$ and let $\mathbf{x}_{1}=\mathbf{f}\left(\mathbf{x}_{0}\right)$. From the inverse function theorem, it follows that

$$
\lim _{\mathbf{x} \rightarrow \mathbf{x}_{1}}\left\|\operatorname{det}\left(\mathrm{Df}^{-1}(\mathbf{x})\right)\right\|=\infty .
$$

Thus, for some $i$ 's in $\{1,2, \ldots, p\}$, the denominator of $\left(\mathbf{f}^{-1}\right)_{i}$ evaluated at $\mathbf{x}_{1}$ is zero. Denote those $i$ by $i_{k}, \quad k=1,2, \ldots, r$. We now prove that at least for one $i_{k}$, num $\left(\left(\mathbf{f}^{-1}\right)_{i_{k}}\right)$ also vanishes at $\mathbf{x}_{1}$. By contradiction, suppose that none of these numerators vanishes at $\mathbf{x}_{1}$. Thus

$$
\lim _{\mathbf{x} \rightarrow \mathbf{x}_{1}}\left\|\mathbf{f}^{-1}(\mathbf{x})\right\|=\infty
$$

On the other hand, we have

$$
\lim _{\mathbf{x} \rightarrow \mathbf{x}_{0}}\left\|\mathbf{f}^{-1}(\mathbf{f}(\mathbf{x}))\right\|=\mathbf{x}_{0},
$$

which contradicts equation (2.29). Thus, $\mathbf{x}_{1}$ must be in one of the following sets

$$
A_{i}=\left\{\mathbf{x} \in \mathbb{C}^{p} \mid \operatorname{num}\left(\left(\mathbf{f}^{-1}\right)_{i}\right)(\mathbf{x})=\operatorname{den}\left(\left(\mathbf{f}^{-1}\right)_{i}\right)(\mathbf{x})=0\right\}, \quad i \in\{1,2, \ldots, p\} .
$$

This concludes the proof since each set $A_{i}$ is defined as the intersection of the zero level sets of two relatively prime polynomials.

As mentioned earlier, the singularity confinement turns out to be closely related to the existence of a first integral. This relationship will be explored in the next section. We define the notion of a first integral.

Definition. A first integral of $\mathbf{x}_{n+1}=\mathbf{f}\left(\mathbf{x}_{n}\right)$ is an analytic function $I: U \subset \mathbb{C}^{p} \rightarrow \mathbb{C}$ where $U$ is a dense subset of $\mathbb{C}^{p}$ such that

(a) $\left\|\left(\frac{\partial I}{\partial x_{1}}, \frac{\partial I}{\partial x_{2}}, \ldots, \frac{\partial I}{\partial x_{p}}\right)\right\| \neq 0$ almost everywhere in $U$.

(b) $I(\mathbf{f}(\mathbf{x}))=I(\mathbf{x})$.

A first integral can exist in several forms.

Definition. A first integral I of $\mathbf{x}_{n+1}=\mathbf{f}\left(\mathbf{x}_{n}\right)$ is polynomial (resp. rational) if the function 
$I(\mathbf{x})$ is a polynomial (resp. rational) function of $\mathbf{x} \in \mathbb{C}^{p}$. A complex-valued function $f(\mathbf{x})$ is algebraic over $\mathbb{C}$ if there exist $s>0$ and $q_{0}, \ldots, q_{s}$ rational in $\mathbf{x}$ such that

$$
q_{0}+q_{1} f+\cdots+q_{s} f^{s}=0 .
$$

If $s$ is the smallest positive integer such that 2.32) holds, the relation (2.32) is referred to as the minimal polynomial of $f$. A first integral $I$ is algebraic if the function $I(\mathbf{x})$ is an algebraic function.

Many notions of integrability are used in the literature. In this paper, we concentrate on algebraic integrability. In order to introduce the latter, we are interested in determining precisely "how many" algebraic first integrals a given discrete system must admit to be algebraically integrable. To further clarify this problem, suppose that the algebraic functions $A_{1}(\mathbf{x}), A_{2}(\mathbf{x}), \ldots, A_{r}(\mathbf{x})$ defined on $\mathbb{C}^{p}$ are all first integrals for the system (2.21) and $F\left(z_{1}, z_{2}, \ldots, z_{r}\right)$ is an algebraic function on $\mathbb{C}^{r}$. Then, it is clear that $A(\mathbf{x})=F\left(A_{1}(\mathbf{x}), A_{2}(\mathbf{x}), \ldots, A_{r}(\mathbf{x})\right)$ is also a first integral. However, this new first integral adds no knowledge to the given problem since it depends on the other first integrals.

Definition. Let $A_{1}(\mathbf{x}), A_{2}(\mathbf{x}), \ldots, A_{r}(\mathbf{x})$ be smooth complex-valued functions defined on a domain $D \subset \mathbb{C}^{p}$. Then

(a) $A_{1}, A_{2}, \ldots, A_{r}$ are functionally dependent if, for each $\mathbf{x} \in D$, there is a neighborhood $U$ of $\mathbf{x}$ and a smooth complex-valued function $F\left(z_{1}, z_{2}, \ldots, z_{r}\right)$ not identically zero on any subset of $\mathbb{C}^{p}$ such that

$$
F\left(A_{1}(\mathbf{x}), A_{2}(\mathbf{x}), \ldots, A_{r}(\mathbf{x})\right)=0 .
$$

(b) $A_{1}, A_{2}, \ldots, A_{r}$ are functionally independent if they are not functionally dependent when restricted to any open subset of $D$.

A simple way to determine if a set of functions are functionally independent is given by the following theorem (see for example [20]).

Theorem. Let $A=\left(A_{1}, A_{2}, \ldots, A_{r}\right)$ be a set of smooth functions from a domain $D \in \mathbb{C}^{p}$ to $\mathbb{C}^{r}$. Then, $A_{1}(\mathbf{x}), A_{2}(\mathbf{x}), \ldots, A_{r}(\mathbf{x})$ are functionally dependent if and only if DA $\left(\mathbf{x}^{*}\right)$ has rank strictly less than $r$ for all $\mathbf{x}^{*} \in D$.

We introduce the notion of integrability considered in this paper.

Definition. The discrete dynamical system $\mathbf{x}_{n+1}=\mathbf{f}\left(\mathbf{x}_{n}\right)$ is said to be algebraically integrable if it admits $(p-1)$ algebraic functionally independent first integrals.

The following theorem is a direct extension of Brun's [6 [19] theorem to discrete systems and is useful to extend the results we obtain for rational first integrals to algebraic ones.

Theorem.: If a discrete dynamical system of $\mathbf{x}_{n+1}=\mathbf{f}\left(\mathbf{x}_{n}\right)$ has $k$ functionally independent algebraic first integrals, then it has $k$ functionally independent rational first integrals.

Proof. Let $I$ be an algebraic first integral and let

$$
P(I)=q_{0}+q_{1} I+\cdots+q_{s-1} I^{s-1}+I^{s}
$$

be its minimal polynomial, where $q_{i}$ is a rational function of $\mathbf{x}$. Since $I$ depends non trivially on $\mathbf{x}$, let $i$ be such that $q_{i}$ is a non constant rational function of $\mathbf{x}$. Since $I(\mathbf{f}(\mathbf{x}))=I(\mathbf{x})$, we have that

$$
q_{0}(\mathbf{f}(\mathbf{x}))-q_{0}(\mathbf{x})+\left(q_{1}(\mathbf{f}(\mathbf{x}))-q_{1}(\mathbf{x})\right) I(\mathbf{x})+\cdots+\left(q_{s-1}(\mathbf{f}(\mathbf{x}))-q_{s-1}(\mathbf{x})\right) I^{s-1}(\mathbf{x})=0 .
$$

Since $P$ is minimal, we have $\left.q_{i}(\mathbf{f}(\mathbf{x}))\right)=q_{i}(\mathbf{x})$ and each $q_{i}$ is a first integral. Now, let $I^{\prime}$ be another independent first integral whose minimal polynomial is

$$
P\left(I^{\prime}\right)=q_{0}^{\prime}+q_{1}^{\prime} I^{\prime}+\cdots+q_{s^{\prime}-1}^{\prime} I^{\prime s^{\prime}-1}+I^{\prime s^{\prime}} .
$$


The independence between the two first integrals implies that there exist $i<s, i^{\prime}<s^{\prime}$ such that $q_{i}, q_{i^{\prime}}$ are two independent non constant rational first integrals. By induction, one can build $k$ independent rational first integrals.

Therefore, rational integrability implies algebraic integrability and it is sufficient to consider discrete dynamical systems of the form (2.21) which admit $(p-1)$ functionally independent rational first integrals $R_{i}(\mathbf{x})=P_{i}(\mathbf{x}) / Q_{i}(\mathbf{x})$ where $i=1,2, \ldots, p-1$ and $P_{i}$ and $Q_{i}$ are relatively prime polynomials. Let $L_{i, c}$ be the level set of the first integral $R_{i}$ corresponding to the value $c_{i} \in \mathbb{C}$. Since $\mathbf{f}$ is continuous, it leaves invariant the closure of the level set given by

$$
\bar{L}_{i, c}=\left\{\mathbf{x} \in \mathbb{C}^{p} \mid P_{i}(\mathbf{x})-c Q_{i}(\mathbf{x})=0\right\} .
$$

\section{The two-dimensional case}

In this section we study the local implications of algebraic integrability on the confinement of singularities for two-dimensional discrete dynamical systems. We consider a discrete dynamical systems of the form (2.21) which is algebraically integrable, that is, it possesses a non trivial rational first integral $R(\mathbf{x})=P(\mathbf{x}) / Q(\mathbf{x})$ where $P$ and $Q$ are some polynomials over $\mathbb{C}^{2}$. Let $L_{c}$ be the level set of $R$ corresponding to the value $c \in \mathbb{C}$. Then, as noted before, $\mathbf{f}$ leaves invariant the closure of the level set $\bar{L}_{c}$.

We first focus our attention on singularities of the first type. The singular set of first type $S_{I}(\mathbf{f})$ is an algebraic variety since it is defined as the set of zeros of polynomials. We consider its irreducible decomposition

$$
S_{I}(\mathbf{f})=\bigcup_{i=1}^{d} S_{I}^{(i)}(\mathbf{f})
$$

where each irreducible component $S_{I}^{(i)}(\mathbf{f})$ is defined as the zero set of an irreducible polynomial on $\mathbb{C}^{2}$.

In Example (2.18), we showed that the existence of a rational first integral does not ensure that all singularities are confined. However, in this case it is not possible to "enter" the singularity, meaning that it does not have a unique preimage under the mapping. Moreover, we know that confinement is a generic property, that is, it is only concerned with dense subsets of irreducible components of $S_{I}$. For instance, in Example (2.15) all singularities of type I of the form $\left(b, y_{0}\right)$ confine in 3 steps except a particular singularity defined by $y_{0}=b /\left(b^{2}-1\right)$. We state the general theorem relating the confinement of singularities to the existence of a rational first integral.

Theorem 3.1. Consider a two-dimensional birational mapping $\mathbf{x}_{n+1}=\mathbf{f}\left(\mathbf{x}_{n}\right)$ and assume it has a rational first integral $R(\mathbf{x})=P(\mathbf{x}) / Q(\mathbf{x})$. Then, for each irreducible component $S_{I}^{(j)}(\mathbf{f})$, we have either

(a) There exists $k_{j} \in \mathbb{N}^{>0}$ such that

$$
\lim _{\mathbf{x} \rightarrow \mathbf{x}^{*}} \mathbf{f}^{k_{j}}(\mathbf{x})
$$

exists for almost all $\mathbf{x}^{*} \in S_{I}^{(j)}(\mathbf{f})$, or,

(b) There exists $l_{j} \in \mathbb{N}^{>0}$ such that $S_{I}^{(j)}(\mathbf{f})$ lies inside the singular set of the birational mapping defining $\mathbf{f}^{-l_{j}}$.

Proof. Without loss of generality, take $j=1$. Consider two cases:

1) $S_{I}^{(1)}(\mathbf{f}) \not \subset \bar{L}_{c}$ for all $c \in \mathbb{C}$.

Let $\mathbf{x}^{*} \in S_{I}^{(1)}(\mathbf{f})$ such that $\lim _{\mathbf{x} \rightarrow \mathbf{x}^{*}}\left\|f_{i}(\mathbf{x})\right\|$ exists or is infinity (as opposed to undefined) for $i=1,2$. Suppose that $\mathbf{x}^{*} \in \bar{L}_{c^{*}}$ for only one $c^{*} \in \mathbb{C}$ and that there is a neighborhood of $\mathbf{x}^{*}$ on $\bar{L}_{c^{*}}$ whose only intersection with any of the singular sets associated with the mappings $\mathbf{f}^{i}$, $i=1,2, \ldots, q+1$ ( $q$ is defined below) is $\mathbf{x}^{*}$ itself. The set of points satisfying the above properties is dense in $S_{I}^{(1)}(\mathbf{f})$. Let $q$ be the number of paths $\mathbf{p}_{i}(\epsilon), i=1,2, . ., q$ in $\mathbb{C}^{2}$ such that 
(i) $\mathbf{p}_{i}(\epsilon) \in \bar{L}_{c^{*}}$ for $\epsilon$ small enough. That is,

$$
P\left(\mathbf{p}_{i}(\epsilon)\right)-c^{*} Q\left(\mathbf{p}_{i}(\epsilon)\right)=0 .
$$

(ii) $\left\|\mathbf{p}_{i}(\epsilon)\right\| \rightarrow \infty$ as $\epsilon \rightarrow 0$ and at least one of the components of $\mathbf{p}_{i}(\epsilon)$ is of the form $1 / \epsilon$.

Note that the number $q$ can depend on the complex number $c^{*}$ but the values it can take for different $c^{*}$ can be bounded above by a number depending only on the degrees of $P$ and $Q$.

Now, consider another path $\mathbf{x}(\sigma) \in \bar{L}_{c^{*}} \forall \sigma$ such that

$$
\lim _{\sigma \rightarrow 0} \mathbf{x}(\sigma)=\mathbf{x}^{*}
$$

Since there is a neighborhood of $\mathbf{x}^{*}$ on $\bar{L}_{c^{*}}$ whose only intersection with $S(\mathbf{f})$ is $\mathbf{x}^{*}, \mathbf{f}(\mathbf{x}(\sigma))$ is well-defined for $\sigma$ nonzero and small enough. Moreover, since $\mathbf{f}$ preserves $\bar{L}_{c^{*}}, \mathbf{f}(\sigma)$ also lies in $\bar{L}_{c^{*}}$, and

$$
\lim _{\sigma \rightarrow 0}\|\mathbf{f}(\mathbf{x}(\sigma))\|=\infty
$$

Therefore, there exists a change of variable $\sigma=\sigma(\epsilon)$ of order $\mathcal{O}\left(\epsilon^{s}\right)$ for some $s>0$, so that $\mathbf{f}(\mathbf{x}(\sigma))$ is one of the paths $\mathbf{p}_{i}$ defined above:

$$
\mathbf{f}(\mathbf{x}(\sigma(\epsilon)))=\mathbf{p}_{i}(\epsilon)
$$

for some $1 \leq i \leq q$. Our claim is that there exists $m$ with $m \leq q+1$ such that the limit as $\sigma \rightarrow 0$ of $\mathbf{f}^{m}(\mathbf{x}(\sigma))$ exists and is finite. Suppose by contradiction that the iterates $\mathbf{f}^{k}(\mathbf{x}(\sigma))$ $k=1,2,3, \ldots, q+1$ are all divergent at $\sigma=0$. Each iterate $\mathbf{f}^{k}(\mathbf{x}(\sigma))$ is associated with a unique path $\mathbf{p}_{i_{k}}(\epsilon)$. This implies that the $k^{\text {th }}$ preimage of $\mathbf{p}_{i_{k}}(\epsilon)$ is finite in the limit $\epsilon \rightarrow 0$ and no other $\mathbf{f}^{l}(\mathbf{x}(\sigma))$ for $1 \leq k<l \leq q+1$ corresponds to the same $\mathbf{p}_{i_{k}}(\epsilon)$. Hence the relation between the $q+1$ iterates that are divergent at $\sigma=0$ and the paths $\mathbf{p}_{i}(\epsilon)$ is injective. This is a contradiction since there are only $q$ paths $\mathbf{p}_{i}(\epsilon)$.

2) There exists a $c \in \mathbb{C}$ such that $S_{I}^{(1)}(\mathbf{f}) \subset \bar{L}_{c}$. Let

$$
\bar{L}_{c}=\bigcup_{i=1}^{r} \bar{L}_{c}^{(i)}
$$

be the irreducible decomposition of $\bar{L}_{c}$ for any $c \in \mathbb{C}$. If $S_{I}^{(1)}(\mathbf{f})$ lies inside the singular set of $\mathbf{f}^{-1}$, then the theorem is proved. Otherwise, let $k \in \mathbb{N}^{>0}$ be such that $S_{I}^{(1)}(\mathbf{f})$ does not lie in a singular set associated with the mappings $\mathbf{f}^{-m}, m=1,2, \ldots, k$. Then,

$$
\overline{\mathbf{f}^{-m}\left(S_{I}^{(1)}(\mathbf{f})\right)}=\bigcup_{i \in B_{m}} \bar{L}_{c}^{(i)}, \quad 1 \leq l \leq k
$$

where each $B_{m}$ is a subset of $\{1,2, \ldots, r\}$. The $k$ sets in (3.8) are distinct, otherwise, by contradiction there exist $m_{1}, m_{2} \in \mathbb{N}^{>0}$ such that

$$
\overline{\mathbf{f}^{-m_{1}}\left(S_{I}^{(1)}(\mathbf{f})\right)}=\overline{\mathbf{f}^{-m_{2}\left(S_{I}^{(1)}(\mathbf{f})\right)}}, \quad 1 \leq m_{1}<m_{2} \leq k .
$$

This implies that

$$
S_{I}^{(1)}(\mathbf{f})=\overline{\mathbf{f}^{-1}\left(\mathbf{f}^{\left(m_{1}-m_{2}+1\right)}\left(S_{I}^{(1)}(\mathbf{f})\right)\right)}
$$

which is a contradiction since $\mathbf{f}$ is undefined almost everywhere on $S_{I}^{(1)}(\mathbf{f})$. Hence the $k$ sets in (3.8) are distinct. However, there are only finitely many possibilities for the sets $B_{m}$ in (3.8). Hence $S_{I}^{(1)}(\mathbf{f})$ must lie inside the singular set of $\mathbf{f}^{-l_{1}}$ for some $l_{1}$. 
Notice that the condition (3.2) does not mean that the singularity is confined since the condition that the Jacobian be nonzero must be satisfied. In order to solve this problem, the singularities of type II have to be considered. The singular set of second type is not, in general, an algebraic variety but its closure

$$
\overline{S_{I I}(\mathbf{f})}=\left\{\mathbf{x}^{*} \in \mathbb{C}^{p} \mid \operatorname{num}\left(\operatorname{det}\left(\operatorname{Df}\left(\mathbf{x}^{*}\right)\right)\right)=0\right\}
$$

is. Hence, it is possible to introduce the irreducible decomposition

$$
\overline{S_{I I}(\mathbf{f})}=\bigcup_{i=1}^{s} \overline{S_{I I}^{(i)}(\mathbf{f})}
$$

where each $S_{I I}^{(i)}(\mathbf{f})$ is a subset of $S_{I I}(\mathbf{f})$. It is now possible to state the general theorem for singularities of second type.

Theorem 3.2. Consider a two-dimensional birational mapping $\mathbf{x}_{n+1}=\mathbf{f}\left(\mathbf{x}_{n}\right)$ and assume it has a rational first integral $R(\mathbf{x})=P(\mathbf{x}) / Q(\mathbf{x})$. Then, for each irreducible component $\overline{S_{I I}^{(j)}(\mathbf{f})}$, we have either

(a) There exists $k_{j} \in \mathbb{N}>0$ such that

$$
\lim _{\mathbf{x} \rightarrow \mathbf{x}^{*}} \operatorname{det}\left(D \mathbf{f}^{\mathbf{k}_{\mathbf{j}}}(\mathbf{x})\right)
$$

exists and is nonzero for almost all $\mathbf{x} \in S_{I I}^{(j)}(\mathbf{f})$, or,

(b) There exists $l_{j} \in \mathbb{N}^{>0}$ such that $S_{I I}^{(j)}(\mathbf{f})$ lies inside the singular set of $\mathbf{f}^{-l_{j}}$.

Proof. The proof is similar to the proof of Theorem 3.1. Again, without loss of generality, we take $j=1$ and consider two cases.

1) $\overline{S_{I I}^{(1)}(\mathbf{f})} \not \subset \bar{L}_{c}$ for all $c \in \mathbb{C}$.

The image of $S_{I I}^{(1)}(\mathbf{f})$ under the mapping $\mathbf{f}$ is a finite subset of $\mathbb{C}^{2}$, thanks to Lemma 2.1. Moreover, $S_{I I}^{(1)}(\mathbf{f})$ is not contained inside any level set. For any point in the image of $S_{I I}^{(1)}(\mathbf{f})$ it is therefore possible to choose two distinct preimages in $S_{I I}^{(1)}(\mathbf{f})$ which lie in two different level sets. Since $\mathbf{f}$ preserves $\bar{L}_{c}$, every point in the image of $S_{I I}^{(1)}(\mathbf{f})$ must lie in the set

$$
\bigcap_{c \in \mathbb{C}} \bar{L}_{c}=\left\{\mathbf{x} \in \mathbb{C}^{2} \mid P(\mathbf{x})=Q(\mathbf{x})=0\right\} .
$$

The elements of this finite set are denoted $\mathbf{s}_{i}, i=1,2, \ldots, m$. Note that in the case of a polynomial first integral, $Q(\mathbf{x})=1$ and this set is empty.

Now, choose $\mathbf{x}^{*} \in S_{I I}^{(1)}(\mathbf{f})$ such that $\mathbf{x}^{*} \in \bar{L}_{c^{*}}$ for only one $c^{*} \in \mathbb{C}$ and there is a neighborhood of $\mathbf{x}^{*}$ on $\bar{L}_{c^{*}}$ whose only intersection with any of the singular sets associated with the mappings $\mathbf{f}^{i}, i=1,2, \ldots, q+r+1$ ( $q$ and $r$ are defined below) is $\mathbf{x}^{*}$ itself. The set of points satisfying the above properties is dense in $S_{I I}^{(1)}(\mathbf{f})$.

Consider the $q$ paths $\mathbf{p}_{i}(\epsilon)$ described in the proof of Theorem 3.1. Consider also the $r$ paths $\mathbf{w}_{i}(\epsilon), i=1,2, . ., r$ in $\mathbb{C}^{2}$ satisfying

(i) $\mathbf{w}_{i}(\epsilon) \in \bar{L}_{c^{*}}$ for $\epsilon$ small enough.

(ii) $\left\|\mathbf{w}_{i}(\epsilon)\right\| \rightarrow \mathbf{s}_{j_{i}}$ as $\epsilon \rightarrow 0$ and one of the components $(k=1$ or 2$)$ of $\mathbf{w}_{i}(\epsilon)$ is of the form $\left(\mathbf{s}_{j_{i}}\right)_{k}+\epsilon$ for some $1 \leq j_{i} \leq m$.

Now, consider another path $\mathbf{x}(\sigma) \in \bar{L}_{c^{*}}$ such that

$$
\lim _{\sigma \rightarrow 0} \mathbf{x}(\sigma)=\mathbf{x}^{*}
$$

Since there is a neighborhood of $\mathbf{x}^{*}$ on $\bar{L}_{c^{*}}$ whose only intersection with $S(\mathbf{f})$ is $\mathbf{x}^{*}, \mathbf{f}(\mathbf{x}(\sigma))$ is well-defined for $\sigma$ nonzero and small enough. Moreover since $\mathbf{f}$ preserves $\bar{L}_{c^{*}}, \mathbf{f}(\mathbf{x}(\sigma)) \in \bar{L}_{c^{*}}$, and

$$
\lim _{\sigma \rightarrow 0} \mathbf{f}(\mathbf{x}(\sigma))=\mathbf{s}_{i}
$$


for some $i$ such that $1 \leq i \leq r$. Following the proof of Theorem 3.1, $\mathbf{f}(\mathbf{x}(\sigma))$ can be associated with a path $\mathbf{w}_{j}(\epsilon)$ (whose limit as $\sigma \rightarrow 0$ is $\mathbf{s}_{i}$ ). Suppose by contradiction that the $d+r+1$ iterates $\mathbf{f}^{k}(\mathbf{x}(\sigma)) k=1,2, \ldots, d+r+1$ are either divergent at $\sigma=0$ or have a limit $\sigma \rightarrow 0$ equal to one of the $\mathbf{s}_{i}$. Each iterate $\mathbf{f}^{k}$ is associated with a path $\mathbf{p}_{i_{k}}(\epsilon)$ or $\mathbf{w}_{i_{k}}(\epsilon)$. This implies that the unique $k^{\text {th }}$ preimage of $\mathbf{p}_{i_{k}}(\epsilon)$ or $\mathbf{w}_{i_{k}}(\epsilon)$ must converge to $\mathbf{x}^{*}$ as $\epsilon \rightarrow 0$. The preimage is unique because there is a neighborhood of $\mathbf{x}^{*}$ on $\bar{L}_{c^{*}}$ whose only intersection with any of the singular sets associated with the mappings $\mathbf{f}^{i}, i=1,2, \ldots, q+r+1$ ( $q$ and $r$ are defined below) is $\mathbf{x}^{*}$ itself. Since $\mathbf{x}^{*}$ is not one of the $\mathbf{s}_{i}$, there cannot be two iterates $\mathbf{f}^{k_{1}}(\mathbf{x}(\sigma))$ and $\mathbf{f}^{k_{2}}(\mathbf{x}(\sigma))$, $k_{1}, k_{2} \leq q+r+1$ associated with the same path. Since there are only $q+r+1$ paths $\mathbf{p}_{i}(\epsilon)$ and $\mathbf{w}_{i}(\epsilon)$, there exists a $k \leq q+r+1$ such that the limit $\sigma \rightarrow 0$ of the $k^{\text {th }}$ iterate of $\mathbf{x}(\sigma)$ must be finite and not equal to one $\mathbf{s}_{i}$ as $\sigma \rightarrow 0$. Therefore, the limit $\sigma \rightarrow 0$ of the Jacobian of $\mathbf{f}^{k_{1}}$ evaluated at $\mathbf{x}(\sigma)$ must be a finite nonvanishing number. This ends the proof in the first case.

2) There exists a $c \in \mathbb{C}$ such that $S_{I}^{(1)}(\mathbf{f}) \subset \bar{L}_{c}$. The argument leading to the conclusion that $S_{I I}^{(j)}(\mathbf{f})$ lies inside the singular set of $\mathbf{f}^{-l_{1}}$ for some $l_{1}$ is identical to the second case considered in the proof of the preceding theorem.

With Theorem 3.2, it is now easy to see that singularities of type I satisfying the condition (3.2) are confined. Indeed, suppose $k_{j}$ is the number considered in the first part of Theorem 3.1. If the Jacobian of $\mathbf{f}^{k_{j}}$ is zero almost everywhere on $S_{I}^{j}(\mathbf{f})$, then $S_{I}^{(j)}(\mathbf{f})$ becomes a subset of $\overline{S_{I I}\left(\mathbf{f}^{k_{j}}\right)}$. According to the proof of Theorem 3.1 we know that $S_{I}^{(j)}(\mathbf{f}) \not \subset \bar{L}_{c}$ for all $c \in \mathbb{C}$. Thus, as a consequence of Theorem 3.2, the singularities in $S_{I}^{(j)}(\mathbf{f})$ must be confined. We can state a general theorem including both types of singularities. To do so, we introduce the irreducible decomposition associated with $S(\mathbf{f})$

$$
S(\mathbf{f})=\bigcup_{i=1}^{d+s} S^{(i)}(\mathbf{f})
$$

where $d$ and $s$ are defined in (3.1) and (3.12).

Theorem 3.3. $\quad$ Consider a two-dimensional birational mapping $\mathbf{x}_{n+1}=\mathbf{f}\left(\mathbf{x}_{n}\right)$ and assume it has a rational first integral $R(\mathbf{x})=P(\mathbf{x}) / Q(\mathbf{x})$. Let $S(\mathbf{f})$ be its singular set with irreducible components $S^{(j)}(\mathbf{f})$. Then, for each $j$, either

(a) Almost all singularities of $S^{(j)}(\mathbf{f})$ are confined in the same number of steps, or,

(b) There exists $l_{j} \in \mathbb{N}^{>0}$ such that $S^{(j)}(\mathbf{f})$ lies inside the singular set of $\mathbf{f}^{-l_{j}}$.

\section{Arbitrary dimensions}

In this section, we extend the result of the previous section to birational mappings of arbitrary dimension $p$. The mappings are assumed to be algebraically integrable, that is, they admit $(p-1)$ functionally independent first integrals $R_{i}(\mathbf{x})=P_{i}(\mathbf{x}) / Q_{i}(\mathbf{x}), i=1,2, \ldots, p-1$. Then, the closure of the level sets associated with $\mathbf{c} \in \mathbb{C}^{p-1}$ is given by

$$
\bar{L}_{\mathbf{c}}=\left\{\mathbf{x} \in \mathbb{C}^{p} \mid P_{i}(\mathbf{x})-c_{i} Q_{i}(\mathbf{x})=0, \text { for } 1 \leq i \leq p-1\right\}=\bigcap_{i=1}^{p-1} \bar{L}_{i, c_{i}},
$$

with $\bar{L}_{i, c_{i}}$ defined in (2.37). A set of constants $\mathbf{c} \in \mathbb{C}^{p-1}$ is said to be regular for the first integrals $\mathbf{R}=\left(R_{1}, R_{2}, \ldots, R_{p-1}\right)$ if $\mathbf{D R}(\mathbf{x})$ has rank equal to $(p-1)$ almost everywhere on $\mathbf{R}^{-1}(\mathbf{c})$. The set $\bar{L}_{\mathbf{c}}$ is then an algebraic variety of codimension $(p-1)$.

Our main Theorems $3.1,3.2$, and 3.3 can be readily generalized to arbitrary dimensions and we only give here an outline of the proofs. 
Theorem 4.1. Consider a p-dimensional birational mapping $\mathbf{x}_{n+1}=\mathbf{f}\left(\mathbf{x}_{n}\right)$ and assume it is algebraically integrable with first integrals $R_{i}(\mathbf{x})=P_{i}(\mathbf{x}) / Q_{i}(\mathbf{x}), i=1,2, \ldots, p-1$. Then for each irreducible component $S_{I}^{(j)}(\mathbf{f})$, we have either

(a) There exists $k_{j} \in \mathbb{N}>0$ such that

$$
\lim _{\mathbf{x} \rightarrow \mathbf{x}^{*}} \mathbf{f}^{k_{j}}(\mathbf{x})
$$

exists for almost all $\mathbf{x} \in S_{I}^{(j)}(\mathbf{f})$, or,

(b) There exists $l_{j} \in \mathbb{N}^{>0}$ such that $S_{I}^{(j)}(\mathbf{f})$ lies inside the singular set of the birational mapping defining $\mathbf{f}^{-l_{j}}$.

Proof. Without loss of generality, take $j=1$. Consider two cases:

1) $S_{I}^{(1)}(\mathbf{f}) \not \subset \bar{L}_{i, c}$ for all $\mathbf{c} \in \mathbb{C}^{p-1}$ and $1 \leq i \leq p-1$.

Let $\mathbf{x}^{*} \in S_{I}^{(1)}(\mathbf{f})$ such that $\lim _{\mathbf{x} \rightarrow \mathbf{x}^{*}}\left\|f_{i}(\mathbf{x})\right\|$ exists or is infinite (as opposed to undefined) for $i=1, \ldots, p$. Suppose that $\mathbf{x}^{*} \in \bar{L}_{\mathbf{c}^{*}}$ for only one $\mathbf{c}^{*} \in \mathbb{C}^{p-1}$ which is regular and that there is a neighborhood of $\mathbf{x}^{*}$ on $\bar{L}_{\mathbf{c}^{*}}$ whose only intersection with the singular sets associated with the mappings $\mathbf{f}^{i}, i=1,2, \ldots, q+1$ ( $q$ is defined below) is $\mathbf{x}^{*}$ itself. The set of points satisfying the above properties is dense in $S_{I}^{(1)}(\mathbf{f})$. Let $q$ be the number of paths $\mathbf{p}_{i}(\epsilon), i=1,2, . ., q$ in $\mathbb{C}^{p}$ such that

(i) $\mathbf{p}_{i}(\epsilon) \in \bar{L}_{\mathbf{c}^{*}}$ for $\epsilon$ small enough. That is,

$$
P_{k}\left(\mathbf{p}_{i}(\epsilon)\right)-c_{k}^{*} Q_{k}\left(\mathbf{p}_{i}(\epsilon)\right)=0, \quad k=1,2, \ldots, p-1 .
$$

(ii) $\left\|\mathbf{p}_{i}(\epsilon)\right\| \rightarrow \infty$ as $\epsilon \rightarrow 0$ and at least one of the components of $\mathbf{p}_{i}(\epsilon)$ is of the form $1 / \epsilon$.

Using the fact that $\bar{L}_{\mathbf{c}^{*}}$ is of codimension $p-1$, the rest of the proof follows exactly the similar case in the proof of Theorem 3.1.

2) There exists a $c \in \mathbb{C}$ and a $1 \leq i \leq p-1$ such that $S_{I}^{(1)}(\mathbf{f}) \subset \bar{L}_{i, c}$.

This part of the proof is identical to the corresponding part in the proof of Theorem 3.1 except that $\bar{L}_{i, c}$ is considered instead of $\bar{L}_{c}$.

Theorem 4.2. $\quad$ Consider a p-dimensional birational mapping $\mathbf{x}_{n+1}=\mathbf{f}\left(\mathbf{x}_{n}\right)$ and assume it is algebraically integrable with rational first integrals $R_{i}(\mathbf{x})=P_{i}(\mathbf{x}) / Q_{i}(\mathbf{x}), i=1,2, \ldots, p-1$. Then, for each irreducible component $\overline{S_{I I}^{(j)}(\mathbf{f})}$, we have either

(a) There exists $k_{j} \in \mathbb{N}^{>0}$ such that

$$
\lim _{\mathbf{x} \rightarrow \mathbf{x}^{*}} \operatorname{det}\left(D \mathbf{f}^{\mathbf{k}_{\mathbf{j}}}(\mathbf{x})\right)
$$

exists and is nonzero for almost all $\mathbf{x} \in S_{I I}^{(j)}(\mathbf{f})$, or,

(b) There exists $l_{j} \in \mathbb{N}^{>0}$ such that $S_{I I}^{(j)}(\mathbf{f})$ lies inside the singular set of $\mathbf{f}^{-l_{j}}$.

Proof. The proof is similar to the proof of Theorem 3.2 and the only difference comes in the first case, when $\overline{S_{I I}^{(1)}(\mathbf{f})} \not \subset \bar{L}_{i, c}$ for all $c \in \mathbb{C}$ and $1 \leq i \leq p-1$. Following the proof of Theorem 3.2, for any point in the image of $S_{I I}^{(1)}(\mathbf{f})$ it is possible to choose two distinct preimages in $S_{I I}^{(1)}(\mathbf{f})$ which lie in two different level sets. Indeed, from Lemma 2.1, the image of $S_{I I}^{(1)}(\mathbf{f})$ under the mapping $\mathbf{f}$ lies in a subset of codimension 2 inside $\mathbb{C}^{p}$. Since $\mathbf{f}$ preserves $\bar{L}_{i, c}$, the points in the image of $S_{I I}^{(1)}(\mathbf{f})$ must lie in the set

$$
\bigcap_{c \in \mathbb{C}, i \leq p-1} \bar{L}_{i, c}=\left\{\mathbf{x} \in \mathbb{C}^{2} \mid P_{i}(\mathbf{x})=Q_{i}(\mathbf{x})=0\right\} .
$$

Since the first integrals are functionally independent, the set defined above is finite. The rest of the proof is similar to the proof of Theorem 3.2 except that one should specify that $\mathbf{c}^{*}$ has to be regular. 
Theorem 4.3. Consider a p-dimensional birational mapping $\mathbf{x}_{n+1}=\mathbf{f}\left(\mathbf{x}_{n}\right)$ and assume it is algebraically integrable. Let $S(\mathbf{f})$ be its singular set with irreducible components $S^{(j)}(\mathbf{f})$. Then, for each $j$, either

(a) Almost all singularities of $S^{(j)}(\mathbf{f})$ are confined in the same number of steps, or,

(b) There exists $l_{j} \in \mathbb{N}^{>0}$ such that $S^{(j)}(\mathbf{f})$ lies inside the singular set of $\mathbf{f}^{-l_{j}}$.

\section{Corollaries and applications}

In the previous two sections, the existence of first integrals was assumed to find local information on the confinement property. Here, the information given by the singularity confinement property is used to obtain global information on the discrete dynamical systems such as the non-existence of algebraic first integral and the degree of possible rational first integrals. The results of this section can be considered as a discrete analog of Yoshida's Theorem [27, 28, 6] which, for ODEs, relates the Kovalevskaya exponents given by the Painlevé test to the degree of a rational first integral. The first corollary is a direct consequence of Theorem 4.3.

Corollary 5.1. Consider a p-dimensional birational mapping $\mathbf{x}_{n+1}=\mathbf{f}\left(\mathbf{x}_{n}\right)$ with a non-empty singular set $S(\mathbf{f})$. If there exists an irreducible components $S^{(j)}(\mathbf{f})$ in which almost all singularities are not confining and such that $S^{(j)}(\mathbf{f}) \not \subset S\left(\mathbf{f}^{-k}\right)$ for any positive $k$, then the system is not algebraically integrable.

As an example of this corollary, we show that System (2.11), defined by

$$
\mathbf{f}(x, y)=\left(\begin{array}{c}
-x-y+a+\frac{b}{x^{3}} \\
x
\end{array}\right)
$$

is not algebraically integrable. Recall that singularities of the form $(0, y)$ are non confining. In order to use Corollary 5.1 and prove that this mapping does not admit an algebraic first integral, one must show that points of the form $(0, y)$ are not generically in the singular set of $\mathbf{f}^{-l}$ for some $l>0$. The singular set of $\mathbf{f}^{-1}$ consists of points of the form $(x, 0)$. Thus, we must prove that generic iterates of $(0, y)$ under $\mathbf{f}^{-1}$ do not belong to the set of points of the form $(x, 0)$. The inverse of the mapping (5.1) is given by

$$
\mathbf{f}^{-1}(x, y)=\left(\begin{array}{c}
y \\
-x-y+a+\frac{b}{y^{3}}
\end{array}\right) .
$$

This mapping can be obtained from $\mathbf{f}$ of (5.1) by interchanging $x$ and $y$. Therefore, we can follow the analysis performed in (2.12) to perform a Laurent expansion in $y$ to show that

$$
\mathbf{f}^{-2}\left(x_{0}, y_{0}\right)=\left(\begin{array}{c}
b / y_{0}^{3}+\left(a-x_{0}\right)-y_{0}+\mathcal{O}\left(y_{0}^{5}\right) \\
-b / y_{0}^{3}+x_{0}+\mathcal{O}\left(y_{0}^{5}\right)
\end{array}\right) .
$$

Moreover, we have

$$
\mathbf{f}^{-3}\left(\begin{array}{l}
n b / y_{0}^{3}+\left(a-x_{0}\right)-y_{0}+\mathcal{O}\left(y_{0}^{5}\right) \\
-n b / y_{0}^{3}+x_{0}+\mathcal{O}\left(y_{0}^{5}\right)
\end{array}\right)=\left(\begin{array}{l}
(n+1) b / y_{0}^{3}+\left(a-x_{0}\right)-y_{0}+\mathcal{O}\left(y_{0}^{5}\right) \\
-(n+1) b / y_{0}^{3}+x_{0}+\mathcal{O}\left(y_{0}^{5}\right)
\end{array}\right),
$$

where $n$ is any positive integer. Hence, points of the form $(0, y)$ are not generically sent to points of the form $(x, 0)$ by applications of $\mathbf{f}^{-1}$ and, from Corollary 5.1, System (2.11) does not admit an algebraic first integral.

In general, algebraic integrability restricts the possible local behavior of singularities. Theorem 4.3 gives two possible local behaviors but in each cases, the singularity can have several different global behaviors. Consider an irreducible component of the singular set $S^{(1)}(\mathbf{f})$. In the proofs of Theorems 3.3 and 4.3, we considered two cases: $S^{(1)}(\mathbf{f}) \not \subset \bar{L}_{\mathbf{c}}$ and $S^{(1)}(\mathbf{f}) \subset \bar{L}_{\mathbf{c}}$. When $S^{(1)}(\mathbf{f}) \not \subset \bar{L}_{\mathbf{c}}$ the singularities of $S_{I}^{(1)}(\mathbf{f})$ are generically confined. In this case, two different types of global behavior can 
be expected. First, after meeting a finite number singularities, the mapping generically never meets other singularities (note that this must also be true in the backward direction, that is for $\mathbf{f}^{-1}$ ). We will referred to such a behavior as global confinement. Second, the singularities may be confined but not globally. Because, for each dynamical system of the form (2.21), there are only finitely many irreducible parts to $S(\mathbf{f})$, the mapping must, at one point, come back to the initial singularity and take $S_{I}^{(1)}(\mathbf{f})$ into itself. We say that such singularities are periodic. We now define formally these two types of global behavior and illustrate them.

Definition. A singularity $\mathbf{x}^{*} \in \mathbb{C}^{p}$ for a birational mapping $\mathbf{x}_{n+1}=\mathbf{f}\left(\mathbf{x}_{n}\right)$ is said to be globally confined if there exist $k^{\prime}, k^{\prime \prime} \in \mathbb{N}^{>0}$ such that $\mathbf{x}^{*} \notin S\left(\mathbf{f}^{k}\right)$ for any $k>k^{\prime}$ and $\mathbf{x}^{*} \notin S\left(\mathbf{f}^{-k}\right)$ for any $k>k^{\prime \prime}$.

An example of such a behavior is given by System (2.2). For this system, points of the form $(x, 0)$ are not sent to points of the form $(0, y)$ through successive applications of $\mathbf{f}$. To prove this, let $\left(x_{n}, y_{n}\right)$ be the $n$th iterate of $(x, 0)$. If $n=4 k$, an expansion of $\mathbf{f}^{4}(x, 0)$ reveals that $\left(x_{4 k}, y_{4 k}\right)=$ $\left(k x+\mathcal{O}\left(x^{2}\right),(-k+1) x+\mathcal{O}\left(x^{2}\right)\right), k=1,2, \ldots$ This fact also implies that $x_{4 k+3}=-k x+\mathcal{O}\left(x^{2}\right)$ and we conclude that $x_{4 k+3}$ and $x_{4 k}$ are neither infinite nor vanishing. By contradiction assume that there exists $n$ such that $\left(x_{n}, y_{n}\right)=(0, y)$. Therefore, either $n=4 k+1$ or $n=4 k+2$. However, from Equation (2.4) we know that $x_{n+2}$ is infinite which is a contradiction. We conclude that no iterate of $(x, 0)$ falls onto a point of the form $(0, y)$ and singularities of the form $(0, y)$ are globally confined.

Definition. Consider the singular set a birational mapping $\mathbf{x}_{n+1}=\mathbf{f}\left(\mathbf{x}_{n}\right)$ and one of its irreducible component $S^{(j)}(\mathbf{f})$. If there is a $k \in \mathbb{N}^{>0}$ such that $\mathbf{f}^{k}\left(S^{(j)}(\mathbf{f})\right)=S^{(j)}(\mathbf{f})$, then the elements of $S^{(j)}(\mathbf{f})$ are said to be periodic singularities of period $k$.

An example of such a behavior is given by a particular case of the Gambier system [13, 14]

$$
\left(\begin{array}{l}
x_{n+1} \\
y_{n+1}
\end{array}\right)=\mathbf{f}\left(x_{n}, y_{n}\right)=\left(\begin{array}{c}
\frac{\left(y_{n}+(1 / a-a-1)\right) x_{n}-a+1}{x_{n}+y_{n}} \\
1-1 / y_{n}
\end{array}\right)
$$

where $a$ is a nonzero complex constant. The system (5.5) admits a singularity of type II at any point of the form $(x, a)$. Then the first iterate of a point of this form is given by $(1 / a-1,1-1 / a)$. But the Jacobians of $\mathbf{f}^{2}$ and $\mathbf{f}^{3}$ at $(x, a)$ are nonzero and the singularity is confined. It is not globally confined since $\mathbf{f}^{4}(x, a)=\mathbf{f}(x, a)=(1 / a-1,1-1 / a)$ and the singularity is periodic. The system admit the following first integral

$$
I(x, y)=\frac{y^{3}-3 y+1}{y(y-1)} .
$$

Now, consider the second case considered in the proofs of Theorems 3.3 and $4.3: S^{(1)}(\mathbf{f}) \subset \bar{L}_{\mathbf{c}}$ for some c. If the singularities of $S^{(1)}(\mathbf{f})$ are generically confined in $k$ steps, then $\mathbf{f}^{k}\left(S^{(1)}(\mathbf{f})\right)$ has to be a set of the form of the RHS of (3.8). Since there are only finitely many of those, the singularities cannot be globally confined. So, either they are periodic, or $S^{(1)}(\mathbf{f})$ lies inside the singular set of $\mathbf{f}^{k}$ for all $k$ greater than a certain $k^{\prime}$. It must also be true in the backward direction and if the singularities are not periodic $S^{(1)}(\mathbf{f})$ lies inside the singular set of $\mathbf{f}^{-k}$ for all $k$ greater than a certain $k^{\prime \prime}$. We will refer to these singularities as ubiquitous singularities. An example of such a behavior is given by the singularities of Equation (2.18) of the form $(x, 0)$. These singularities are not confined but they are in the singular set of $\mathbf{f}^{-l}$ for any $l>0$ and therefore, these singularities are ubiquitous. Note that singularities of the form $(0, y)$ are also ubiquitous.

Definition. A singularity $\mathbf{x}^{*} \in \mathbb{C}^{p}$ of a birational mapping is said to be ubiquitous if there exist $a k^{\prime} \in \mathbb{N}^{>0}$ such that $\mathbf{x}^{*} \in S\left(\mathbf{f}^{k}\right)$ for any $k>k^{\prime}$ and $a k^{\prime \prime} \in \mathbb{N}^{>0}$ such that $\mathbf{x}^{*} \in S\left(\mathbf{f}^{-k}\right)$ for any $k>k^{\prime \prime}$. 
Given a discrete dynamical system of the form (2.21) and an irreducible component $S^{(j)}(\mathbf{f})$ of the singular set, one would like to be able to obtain information on the first integrals. The only way one can know if a given irreducible component of the singular set lies inside a level set is by studying the global behavior of the singularity. If the singularity is globally confined, then $S^{(j)}(\mathbf{f})$ does not lie inside a level set, but if it is ubiquitous, then it does. If the singularities are periodic, one cannot reach a conclusion.

Information on first integrals can be obtained by studying singularities of type I. Consider the paths $\mathbf{p}_{i}$ defined in the 2-dimensional case in the proof of Theorem 3.1. The number of these paths is bounded above by the maximum of the degrees of $P$ and $Q$. Moreover, for each $S_{I}^{(j)}(\mathbf{f}) \not \subset \bar{L}_{c}$ for all $c$, consider $k_{j}^{\prime}$, the lowest value $k_{j}$ can take. Then $k_{j}^{\prime}-1$ is the number of different paths $\mathbf{p}_{i}$ realized by iterating a point close to the singularity under the mapping. Hence, the following corollary holds.

Corollary 5.2. Consider a 2-dimensional birational mapping with rational first integral $R=P / Q$. Consider also the irreducible components of the singular set of the first type $S_{I}^{(j)}(\mathbf{f})$ for which the singularities are globally confined. Then the maximum of the degrees of $P$ and $Q$ is bounded below by the number

$$
\sum_{j}\left(k_{j}^{\prime}-1\right)
$$

where $k_{j}^{\prime}$ is given by the lowest value $k_{j}$ of Theorem 3.1.

In Example (2.2), the singular set has a unique component in which singularities are globally confined with $k=4$ and the degree of the first integral is exactly $k-1=3$. Note, however, that the corollary only provides a lower bound as shown in the following example

$$
\left(\begin{array}{c}
x_{n+1} \\
y_{n+1}
\end{array}\right)=\mathbf{f}\left(x_{n}, y_{n}\right)=\left(\begin{array}{c}
-y_{n}+x_{n}+\frac{a}{x_{n}} \\
x_{n}
\end{array}\right),
$$

where $a$ is a non-vanishing complex number. Here again, one can check that singularities of the form $(0, y)$ are confined in 4 steps and since confinement is global, the lower bound for the numerator and denominator of a first integral is 3. However, the rational first integral $I=P / Q$ with lowest degree possible on $P$ and $Q$ is given by

$$
I=\left(x_{n}-y_{n}\right)^{2}\left(x_{n} y_{n}-a\right)^{2},
$$

which is of degree 6 , not 3 (note however the rather degenerate nature of the first integral).

Next, we turn our attention to singularities of type II in the case when $S_{I I}^{(j)}(\mathbf{f}) \not \subset \bar{L}_{\mathbf{c}}$ for all c. From the proof of Theorems 3.2 and 4.2, one sees that, as long as the singularities are not confined, the iterates of $S_{I I}^{(j)}(\mathbf{f}) \not \subset \bar{L}_{\mathbf{c}}$ must be roots of both $P_{i}$ and $Q_{i}$ for all rational first integrals $P_{i} / Q_{i}$. Therefore, we have the following corollary.

Corollary 5.3. $\quad$ Consider an algebraically integrable p-dimensional birational mapping with the rational first integral $R=P / Q$. Consider also an irreducible component of the singular set of the second type $S_{I I}^{(j)}(\mathbf{f})$ whose singularities are globally confined. Let $k_{j}$ be the corresponding confinement number. Then the finite elements of the set

$$
\bigcup_{i=1}^{k_{j}-1} \mathbf{f}^{i}\left(S_{I}^{(j)}(\mathbf{f})\right)
$$

are roots of both $P$ and $Q$.

This corollary is best illustrated on Example (2.15) where $(0, a)$ and $(1 / a, 0)$ are roots of both the numerator and denominator of the rational function (2.17). 
The previous corollary has an immediate consequence for the existence of polynomial first integrals.

Corollary 5.4. Consider an algebraically integrable p-dimensional birational mapping. If this system admits singularities of the second type which are globally confined, then none of the first integrals are polynomial.

The last result concerns the case when the irreducible component of the singular set lies inside the closure of a level set of the first integral. In Sections 3 and 4 , it was found that if $\mathbf{f}^{k}$ (for $k \in \mathbb{N}$ ) is well-defined on $S^{(j)}(\mathbf{f})$ and its Jacobian is nonzero, then $\mathbf{f}^{k}\left(S^{(j)}(\mathbf{f})\right)$ must be a set of the form of the RHS of (3.8), hence it lies on a level set. This is stated in a following corollary.

Corollary 5.5. Consider a p-dimensional birational mapping $\mathbf{x}_{n+1}=\mathbf{f}\left(\mathbf{x}_{n}\right)$ together with an irreducible component of the singular set $S^{(j)}(\mathbf{f})$ for which the singularities are ubiquitous. If $\mathbf{f}^{k}$ is well-defined almost everywhere on $S^{(j)}(\mathbf{f})$ and its Jacobian is nonzero, then $\mathbf{f}^{k}\left(S^{(j)}(\mathbf{f})\right)$ lies inside the closure of a level set of the first integrals. Note that the case $k=0$ should also be included meaning that $S^{(j)}(\mathbf{f})$ itself lies inside the closure of a level set of the first integrals.

This corollary can be applied to Example (2.18). Since the two sets of singularities $((x, 0)$ and $(0, y))$ are ubiquitous, they are level sets of the first integral (2.19).

As a final example, consider the system

$$
\left(\begin{array}{l}
x_{n+1} \\
y_{n+1}
\end{array}\right)=\mathbf{f}\left(x_{n}, y_{n}\right)=\left(\begin{array}{c}
-y_{n}+x_{n}+\frac{a}{x_{n}^{2}} \\
x_{n}
\end{array}\right),
$$

where $a$ is a nonzero complex number. This example was first used in [16] to show that singularity confinement is not sufficient for integrability. Following Example (2.2), it is easy to show that singularities of the form $(0, y)$ are generically globally confined. However, numerical analysis performed on this system strongly suggest that is exhibits chaotic behavior [16.

\section{Conclusions}

In this paper, we defined different types of singularities and their confinement property for autonomous discrete birational dynamical systems. The theory of singularity confinement was developed more than 10 years ago [11,23, 12, and different types of singularities have been implicitly used. Here, in order to relate algebraic integrability to singularity confinement we have found necessary to give a formal definition of different singularities and their confinement property.

In Sections III and IV, we showed that algebraic integrability for birational mappings imply that singularities are either locally confined or some preimages are not well-defined.

In Section V, we studied global properties of birational mappings and showed that there are three types of behavior for singularities compatible with algebraic integrability. Namely, singularities are either globally confined, periodic, or ubiquitous. Remarkably, in the seminal papers describing singularity confinement, similar behaviors were found heuristically to be compatible with integrability [11, 23, 12, 25, 13, 15. However, the classical approach relies mostly on a local analysis of the singularities. An important aspect of the results shown in this paper is that global properties have to be satisfied for a system to be algebraically integrable. To unify these different concepts, we define the confinement property for a discrete system in the following way:

Definition. A birational mapping is said to have the confinement property if its singularities are, generically, globally confined, periodic, or ubiquitous.

Then the most important result of this paper can be rephrased as follows.

Theorem. An algebraically integrable birational mapping has the confinement property. 
In particular, this result shows that every mapping in the QRT-family 22, 15 has the confinement property both in the symmetric and asymmetric cases. This fact also holds for the recently discovered class of integrable birational discrete systems discovered in [18. Moreover, from examples such as System (5.10), we have strong numerical evidence that the confinement property is necessary but not sufficient for algebraic integrability.

In this paper, we also found sufficient conditions for non-integrability. Essentially, if singularities are confined in one direction and not in the other, the system cannot be algebraically integrable.

Finally, the remaining corollaries of Section 5 provide information on the existence and degree of rational first integrals directly from the singularity confinement procedure.

All the examples of integrable discrete systems considered in this article have a first integral of genus 0 or 1 . It is not possible to find a birational mapping of infinite order preserving an algebraic curve of genus 2 or higher. The argument proving this fact is due to Veselov ( [29], page 35). It makes use of Hurwitz theorem which tells us that the automorphic group of an algebraic curve of genus 2 or higher is finite.

Since the only mappings studied here were birational, we could have made use of the projective space in which the point at infinity plays no particular role. Hence only singularities of type II should be studied. However, the ideas presented in this paper could not be extended for the case of nonautonomous or for discrete dynamical systems that are not birational. For instance, the integrable non rational mapping derived in 89 10] could not be studied. It is therefore crucial to give a definition of singularity confinement that can be applied to general discrete systems.

Another important question concerns the relation between the confinement property and other types of integrability for discrete dynamical systems (see for example [2,3,4,16,24]).

\section{Acknowledgment}

A.G. acknowledges a Fellowship from the Sloan Foundation. S.L. acknowledges a post-doctoral fellowship from NSERC (National Science and Engineering Research Council of Canada). The authors are grateful to Basil Grammaticos, Alfred Ramani and John Roberts for helpful discussions.

\section{References}

[1] M.J. Ablowitz, A. Ramani, H. Segur, Nonlinear evolution equations and ordinary differential equations of Painlevé type, Lettere al Nuovo Cimento 23, 333-338 (1978).

[2] M.J. Ablowitz, R. Halburd and B. Herbst, On the extension of the Painlevé property to difference equations, Nonlinearity 13, 889-905 (2000).

[3] M.P. Bellon and C.-M. Viallet, Algebraic entropy, Comm. Math. Phys. 204, 425-437 (1999).

[4] R. Conte and M. Musette, A new method to test discrete Painlevé equations, Phys. Lett. A 223, 439-448 (1996).

[5] B. Gambier, Sur les équations différentielles du second ordre et du premier degré dont l'intégrale générale est à points critiques fixes, Acta Math. 33, 1-55 (1910).

[6] A. Goriely, Integrability and nonintegrability of dynamical systems, World Scientific, Singapore 2001.

[7] A. Goriely, Integrability, partial integrability, and nonintegrability for systems of ordinary differential equations, J. Math. Phys. 37, 1871-1893 (1996).

[8] A. Iatrou and J.A.G. Roberts, Integrable Mappings of the plane preserving biquadratic invariant curves, J. Phys. A 34, 6617-6636 (2001).

[9] A. Iatrou and J.A.G. Roberts, Integrable Mappings of the plane preserving biquadratic invariant curves II, Nonlinearity 15, 459-489 (2002).

[10] A. Iatrou and J.A.G. Roberts, Integrable Mappings of the plane preserving biquadratic invariant curves III, Physica A 326, 400-411 (2003).

[11] B. Grammaticos, A. Ramani and V.G. Papageorgiou, Do integrable mappings have the Painlevé property?, Phys. Rev. Lett. 67, 1825-1826 (1991). 
[12] B. Grammaticos, A. Ramani and K.M. Tamizhmani, Nonproliferation of pre-images in integrable mappings, J. Phys A 27, 559-566 (1994).

[13] B. Grammaticos and A. Ramani, The Gambier mapping, Physica A 223, 125-136 (1995).

[14] B. Grammaticos, A. Ramani and S. Lafortune The Gambier mapping, revisited, Physica A 253, 260-270 (1998).

[15] B. Grammaticos, F. Nijhoff and A. Ramani, Discrete Painlevé equations. In: R. Conte (ed.) The Painlevé property. Proceedings, Cargese 1996, pp. 413-516. New-York: CRM Ser. Math. Phys., Springer, 1999.

[16] J. Hietarinta and C.-M. Viallet, Singularity confinement and chaos in discrete systems, Phys. Rev. Lett. 81, 325-328 (1998).

[17] E.L. Ince, Ordinary differential equations. New-York: Dover, 1956.

[18] K. Kimura, H. Yahagi, R. Hirota, A. Ramani, B. Grammaticos and Y. Ohta, A new class of integrable discrete systems, J. Phys. A 35, 9205-9212 (2002).

[19] Kummer, M., R. C. Churchill, and D. L. Rod, On a result of Brun, Can. Math. Bull 33, 175-180 (1990).

[20] P. J., Olver, Applications of Lie groups to differential equations. New-York: Springer-Verlag, 1993.

[21] P. Painlevé, Oeuvres de Paul Painlevé. Tome I. Paris: Editions du CNRS, 1973.

[22] G.R.W. Quispel, J.A.G. Roberts and C.J. Thompson, Integrable mappings and soliton equations II, Physica D 34, 183-192 (1989).

[23] A. Ramani, B. Grammaticos and J. Hietarinta Discrete versions of the Painlevé equations, Phys. Rev. Lett. 67, 1829-1832 (1991).

[24] J.A.G. Roberts, D. Jogia and F. Vivaldi, The Hasse-Weil Bound and Integrability Detection in Rational Maps, J. Nonl. Math. Phys. (in press).

[25] A. Ramani, B. Grammaticos and G. Karra, Linearizable mappings, Physica A 180, 115-127 (1992).

[26] A. Ramani, B. Grammaticos, K.M. Tamizhmani and S. Lafortune, Again, linearizable mappings, Physica A 252, 138-150 (1998).

[27] Yoshida, H., Necessary conditions for the existence of algebraic first integrals I. Celest. Mech., 31, 363-379 (1983).

[28] Yoshida, H., Necessary conditions for the existence of algebraic first integrals II. Celest. Mech., 31, 381-399 (1983).

[29] A.P. Veselov, Integrable maps, Russ. Math. Survey46, 1-51 (1991). 